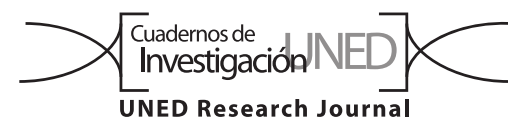

\title{
Respuestas morfogenéticas de plantas in vitro y esquejes de Vanilla planifolia (Orchidaceae) durante el desarrollo inicial del cultivo en invernadero y en sistemas agroforestales
}

\author{
José Bernal Azofeifa-Bolaños ${ }^{1,3}$, German Rivera-Coto², Amelia Paniagua-Vásquez ${ }^{3}$ \\ \& Roberto Cordero-Solórzano ${ }^{4}$ \\ 1. Maestría en Manejo de Recursos Naturales, Universidad Estatal a Distancia, San José, Costa Rica; jb12azo@gmail.com \\ 2. Escuela de Ciencias Agrarias, Universidad Nacional de Costa Rica, Heredia, Costa Rica. \\ 3. Instituto de Investigación y Servicios Forestales, Universidad Nacional de Costa Rica, Heredia, Costa Rica. \\ 4. Escuela de Ciencias Biológicas, Universidad Nacional de Costa Rica, Heredia, Costa Rica.
}

Recibido 23-I-2018 • Corregido 09-IV-2018 • Aceptado 23-IV-2018

\begin{abstract}
Morphogenetic responses of cuttings and in vitro plants of Vanilla planifolia (Orchidaceae) during initial development in greenhouse and agroforestry systems. There is little knowledge in Costa Rica about morphogenetic responses of Vanilla planifolia. Here we compare cutting and micro-cutting for morphogenetic response in nursery and agroforestry. Each plant type was grown in both conditions and the combination with factor levels resulted in four treatments. The independent growth responses of the cuttings were significantly higher for the length, total weight and emergent shoot, total and average length and weight of the roots compared to that in micro-cuttings. For this factor, a significant increase of nodes was achieved when micro-cuttings were used. The length, total weight and emergent shoot, weight and number of roots of the explants grown in the field were significantly greater compared to that in nursery. Total and average length of the roots did not significantly differ between independent treatments of condition factor. Significant interaction between factors were obtained for all variables except for the total weight and the emergent shoot. After a period of nursery acclimatization, the agroforestry vitroplants were significantly higher for the length of the shoot, number of nodes and number of roots in relation to the cuttings grown in both conditions, as well as the vitroplants grown in nursery. Longer and heavier roots were obtained when cuttings were grown at the nursery, suggesting a more intense growth in the search for water and nutrients.
\end{abstract}

Key words: vanilla, wild type relatives, domestication, Costa Rica.

En los últimos 30 años, el conocimiento en Costa Rica sobre aspectos morfogenéticos en vainilla, tanto en condiciones de invernadero y de campo, se debe, casi exclusivamente, al cultivo de un híbrido desarrollado en Madagascar entre Vanilla planifolia (herencia materna) y
RESUMEN: El conocimiento de las respuestas morfogenéticas de Vanilla planifolia en Costa Rica es muy limitado. En esta investigación comparamos las respuestas morfogenéticas de esquejes convencionales y plántulas in vitro en invernadero y en un sistema agroforestal. Cada tipo de explante se cultivó en ambas condiciones de cultivo y la combinación de los niveles de cada factor resultó en cuatro tratamientos. Las respuestas de crecimiento independientes de los esquejes fueron significativamente mayores para la longitud, peso total y del brote emergente, longitud total, promedio y peso de las raíces en comparación con las plántulas in vitro. Para este factor, se obtuvo de manera significativa más nudos cuando se usaron plántulas provenientes del cultivo in vitro. Los explantes cultivados en campo obtuvieron diferencias significativamente superiores para la longitud, peso total y del brote emergente, peso y número de raíces en relación con los cultivados en vivero. Sin embargo, la longitud total y promedio de las raíces no difirió de manera significativa entre los tratamientos independientes del factor condición. Se obtuvieron interacciones significativas entre los factores para todas las variables con excepción del peso total y del brote emergente. Después de un periodo de aclimatización en invernadero, las vitroplantas cultivadas en el sistema agroforestal fueron significativamente más largas, con más nudos y raíces en comparación con los esquejes cultivados en ambas condiciones, así como, las vitroplantas cultivadas en invernadero. Se obtuvieron raíces más largas y pesadas cuando los esquejes se cultivaron en invernadero, lo cual sugiere un crecimiento más intenso en la búsqueda de agua y nutrientes.

Palabras claves: vainilla, parientes silvestres, domesticación, Costa Rica. 
consumidor mundial) como productos alimenticios (21CFR169.175).

Para lograr el ingreso a ese mercado en condiciones óptimas de competencia es necesario un cambio en las poblaciones de vainilla cultivada en el país, para lo cual se requiere contar con información básica sobre una serie de aspectos agronómicos que garanticen el éxito de futuras plantaciones. Cuando se analiza la disponibilidad de tales conocimientos generados en Costa Rica, se observa un vacío de información aplicable a V. planifolia. Esta especie, de la cual hay materiales silvestres en el país, puede establecerse por dos vías: por medio de plantas producidas in vitro o mediante esquejes obtenidos en fincas o de plantas silvestres. La primera opción es la más viable, pues no pone en riesgo las escasas poblaciones silvestres (Azofeifa-Bolaños, Paniagua-Vásquez \& García-García, 2014). Sin embargo, no se cuenta con parámetros comparativos de la respuesta morfogenética de plantas producidas in vitro respecto a esquejes de plantas, durante el desarrollo inicial en invernadero y campo; información fundamental para promover el uso y comercialización de recursos fitogenéticos nativos, sin afectar su conservación, dándoles un manejo sostenible en el tiempo. Lo cual se enmarca dentro del modelo de desarrollo impulsado por el gobierno, que busca la conciliación de la conservación del patrimonio natural con el crecimiento económico (Azofeifa-Bolaños et al., 2014). Consciente de esta problemática la Universidad Nacional de Costa Rica, está realizando esfuerzos para propiciar este modelo dentro de sus investigaciones, por lo que procura dar el mejor manejo posible al material genético de vainilla que posee en el banco de germoplasma, que entre sus accesiones cuenta con diez genotipos silvestres de $V$. planifolia coleccionados en los bosques de la costa Caribe (Azofeifa-Bolaños et al., 2017). Este germoplasma es de importancia para preservar e incrementar la variabilidad genética del cultivo, debido a las condiciones críticas de conservación de la especie en estado silvestre (Soto-Arenas, 1999; Soto-Arenas \& Solano-Gómez, 2007). Como parte de esta estrategia, es prioritario la caracterización agronómica de este material silvestre para generar datos de su potencial productivo que permita la toma de decisiones por parte de los mejoradores del cultivo.

Por lo tanto, el beneficio principal de este estudio será determinar si las plantas procedentes de laboratorio, muestran características agronómicas superiores durante el desarrollo inicial del cultivo, con respecto a las procedentes de esquejes de plantas maduras originarias de poblaciones silvestres.

\section{MATERIALES Y MÉTODOS}

Los explantes usados para el experimento fueron obtenidos de plantas de V. planifolia caracterizadas mediante descriptores morfológicos y moleculares correspondientes a individuos de la accesión UNA-VAN-00229 de la colección de vainilla a cargo de la UNA (AzofeifaBolaños et al., 2017).

La aclimatización de las vitroplantas y los ensayos de vivero se realizaron en un invernadero del Instituto de Investigación y Servicios Forestales (INISEFORUNA), Santa Lucía, Barva, Heredia $\left(10^{\circ} 01^{\prime} 21,93^{\prime \prime} \mathrm{N}\right.$ $\left.84^{\circ} 06^{\prime} 43,6^{\prime \prime} \mathrm{W}\right)$, el cual fue acondicionado con sarán (malla al 50\% de sombra). Las condiciones atmosféricas dentro del invernadero tuvieron un promedio de $81 \%$ de humedad relativa con valores mínimos de $38,5 \%$ y máximos de $100 \%$ y una temperatura de $23^{\circ} \mathrm{C}$ que osciló entre 17 y $39^{\circ} \mathrm{C}$. Se aplicó riego manual dos veces por semana.

La investigación en campo se realizó en la finca "Río Blanco", ubicada en La Colonia de Guápiles, Pococí, Limón (10¹5'57,1" $\left.\mathrm{N}-83^{\circ} 49^{\prime} 06,4^{\prime \prime} \mathrm{W}\right)$ (Fig. 1). La parcela experimental de $5239 \mathrm{~m}^{2}$ se trabajó bajo un diseño espacial en sistemas agroforestales con árboles de las especies Minquartia guianensis Aubl., Cordia alliodora (Ruiz \& Pav.) Oken., Virola koschnyi Warb., Vochysia ferruginea Mart. e Hyeronima alchorneoides Allemão sembrados cada $5 \times 5 \mathrm{~m}$ y árboles tutores de las especies Erythrina sp (Poró) y Gliricidia sepium (madero negro) sembrados cada $2 \times 2 \mathrm{~m}$, los cuales le proporcionaron sombra natural a los explantes de vainilla (Fig. 2). La topografía de la zona es plana con una altura promedio de 157 m.s.n.m. El clima es cálido húmedo con promedios anuales de 4 $577,2 \mathrm{~mm}$ de precipitación, $87 \%$ de humedad relativa y $24,4^{\circ} \mathrm{C}$ de temperatura (IMN, 2011). Los suelos de la zona son franco arenosos. La zona de vida según Holdridge es bosque muy húmedo tropical (bmhT) (Quesada, 2007).

Con el objetivo de comparar el desempeño agronómico inicial de esquejes y vitroplantas durante el establecimiento en invernadero y campo, se realizó un experimento bifactorial (2x2). El primer factor consistió en el tipo de explante a dos niveles: esqueje tradicional y vitroplanta. El segundo factor fue la condición de cultivo, invernadero y campo. La combinación de los niveles de ambos factores resultó en cuatro tratamientos (Fig. 3).

En el invernadero, cada tipo de explante se cultivó en un sustrato compuesto de una capa de piedra y arena en proporciones iguales, con musgo Sphagnum L. sobre la parte basal de cada planta, asociado a tutores artificiales de madera, mientras en la condición de campo, los 


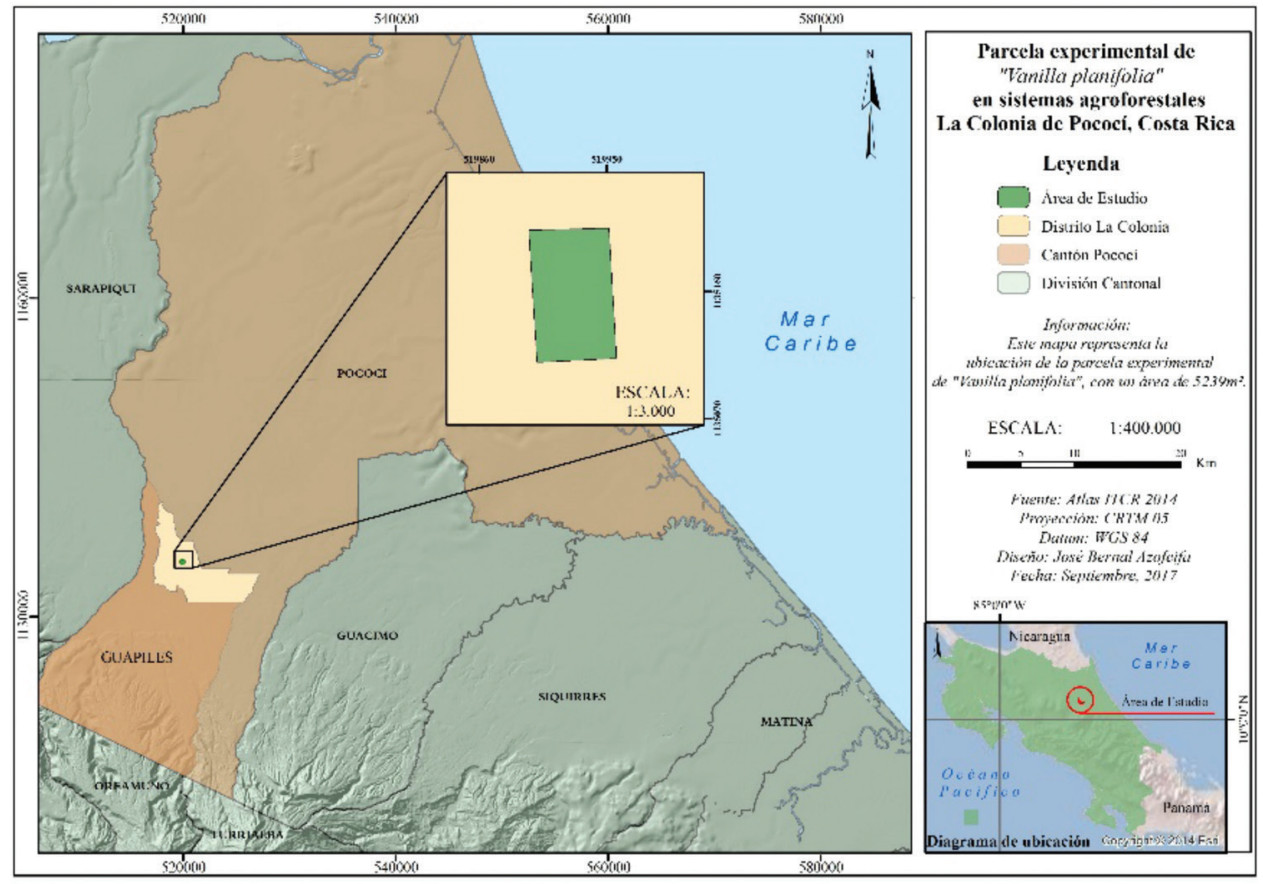

Fig. 1. Ubicación de la parcela experimental de Vanilla planifolia en sistemas agroforestales, Guápiles, Costa Rica.

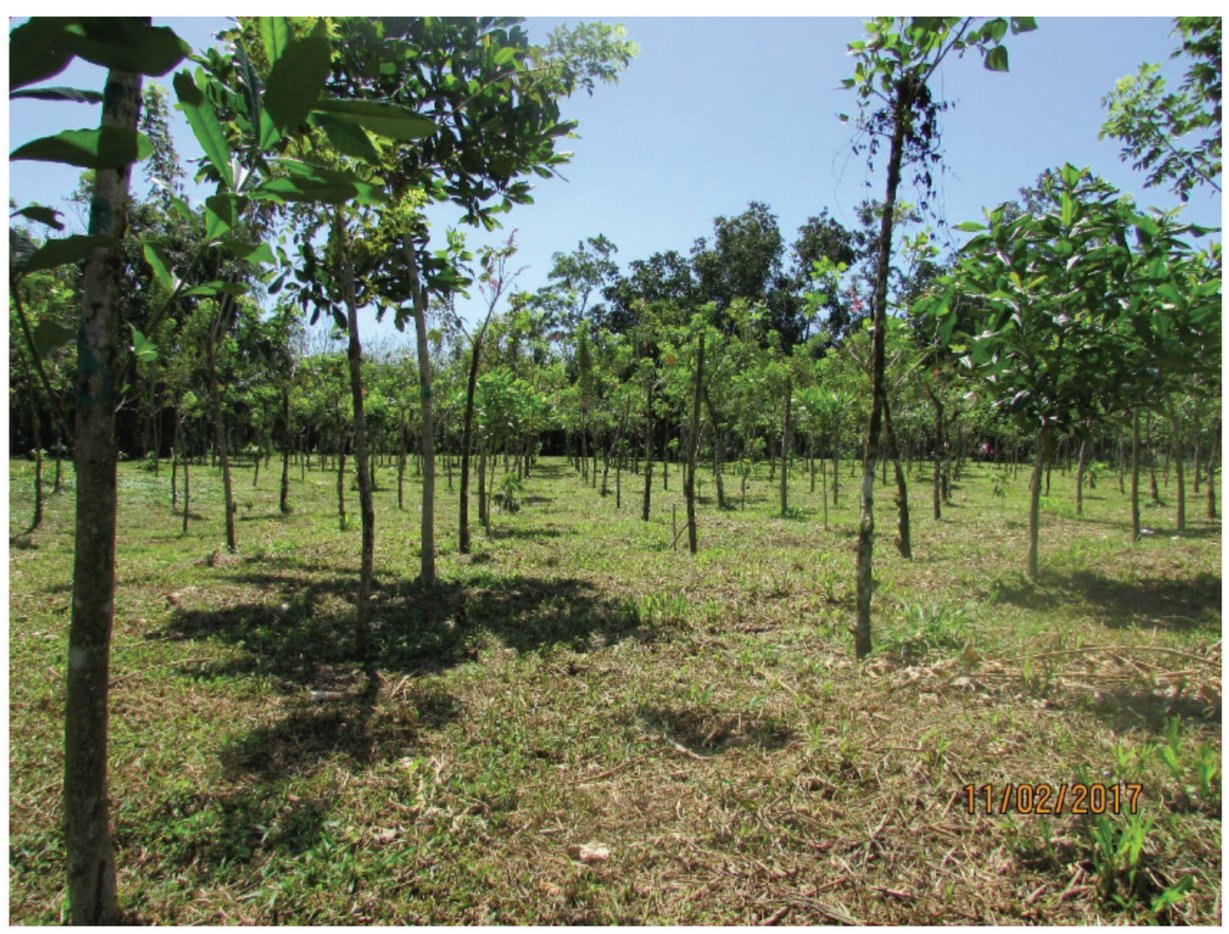

Fig. 2. Parcela experimental establecida en La Colonia de Guápiles con un arreglo espacial en sistemas agroforestales. En la foto, árboles de Vochysia sp. y Erythrina sp. 


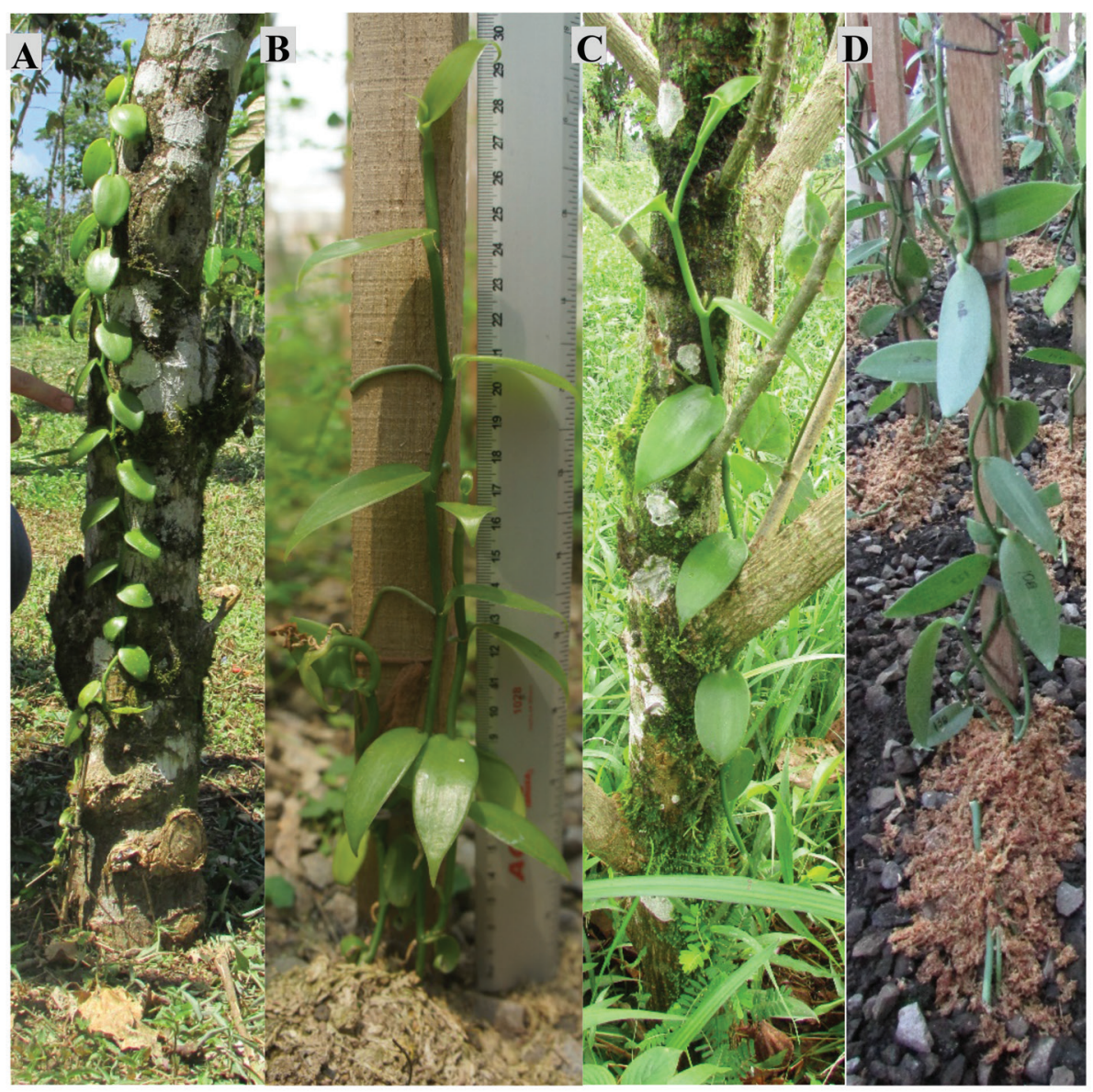

Fig. 3. Tipos de explantes en cada condición de crecimiento: (A) vitroplanta en campo y (B) en invernadero. (C) Esqueje en campo y (D) en invernadero.

explantes se cultivaron en asocio a un árbol tutor de Poró (Erythrina sp.) con una longitud aproximada de 2,0m.

Se utilizó un diseño experimental no balanceado completamente al azar con 49 repeticiones para el tratamiento que usó esquejes y 41 para el tratamiento que usó vitroplantas. La unidad experimental consistió de un tipo de explante con ocho nudos cultivada en cada condición. Para evitar heterogeneidad en los resultados, las plantas usadas fueron uniformes en longitud y peso (Gómez, 2012). Para el caso de los esquejes, se utilizaron un total de 69 unidades experimentales con una longitud promedio de $61,02 \mathrm{~cm}$ y un peso de $20,7 \mathrm{~g}$. Por su parte, se utilizaron 41 vitroplantas con una longitud aproximada de $18,26 \mathrm{~cm}$ y un peso promedio de $3,26 \mathrm{~g}$. En total se escogieron y cultivaron 90 tipos de explantes en ambas condiciones durante seis meses.

Después de este periodo, se midió la longitud, el peso total y del brote nuevo, el número de nudos; la longitud total y promedio, el peso y el número de raíces totales.
Los datos obtenidos fueron sometidos a un análisis de varianza previa comprobación de la normalidad de los datos y homogeneidad de varianzas. Todos los análisis estadísticos se realizaron con el programa SAS v9.4 (SAS Institute Inc., Cary, NC, USA). Para los casos donde se registró diferencias significativas entre los niveles de un factor, se realizó la separación de las medias de mínimos cuadrados mediante la prueba DMS protegida ("protected LSD" en inglés).

\section{RESULTADOS}

En relación con la respuesta morfogenética de los explantes, se presentaron diferencias significativas $(p \leq 0,032)$ entre los tratamientos que evaluaron el tipo de explante y entre las condiciones de cultivo para las variables longitud, peso total y del brote emergente, número de nudos y peso de las raíces (Cuadro 1 y 2). 
CUADRO 1

Medias de algunas variables de crecimiento ( \pm error estándar) de los brotes de $V$. planifolia según el tipo de explante

\begin{tabular}{|c|c|c|c|c|c|c|c|c|}
\hline \multirow{2}{*}{ Tratamiento } & \multicolumn{4}{|c|}{ Brote } & \multicolumn{4}{|c|}{ Raíces } \\
\hline & Longitud (cm) & Peso Total (g) & Peso (g) & Número nudos & Longitud total $(\mathrm{cm})$ & Longitud $(\mathrm{cm})$ & Peso (g) & Número \\
\hline In vitro $(\mathrm{n}=41)$ & $37,01(2,62) a$ & $11,61(1,93) \mathrm{a}$ & $9,85(1,30) a$ & $14,07(0,62) b$ & $80,16(8,83) a$ & $5,40(0,58) a$ & $0,96(0,17) a$ & $12,45(0,63) a$ \\
\hline \multirow[t]{3}{*}{ Esqueje $(n=49)$} & $44,82(2,44) b$ & $32,03(1,80) b$ & $18,18(1,24) b$ & $11,17(0,57) a$ & $132,54(8,22) \mathrm{b}$ & $10,92(0,54) b$ & $2,11(0,15) b$ & $12,01(0,59) \mathrm{a}$ \\
\hline & $F^{*}(p)^{* *}$ & $F(p)$ & $F(p)$ & $F(p)$ & $F(p)$ & $F(p)$ & $F(p)$ & $F(p)$ \\
\hline & $4,75(\mathbf{0}, \mathbf{0 3 2})$ & $59,76(<, 0001)$ & $21,48(<, 0001)$ & $11,88(\mathbf{0}, 0009)$ & $18,85(<, 0001)$ & $48,48(<, 0001)$ & $25,78(<, 0001)$ & $0,26(0,6117)$ \\
\hline
\end{tabular}

*Estadístico $F$. **Probabilidad asociada al ANDEVA, en negrita los valores significativos. Tratamientos cuyas medias tienen letras diferentes en una misma columna difieren $(p<0,05)$ según el método de medias de mínimos cuadrados.

CUADRO 2

Medias de algunas variables de crecimiento ( \pm error estándar) de los brotes de V. planifolia según la condición de cultivo

\begin{tabular}{|c|c|c|c|c|c|c|c|c|}
\hline \multirow{2}{*}{ Tratamiento } & \multicolumn{4}{|c|}{ Brote } & \multicolumn{4}{|c|}{ Raíces } \\
\hline & Longitud $(\mathrm{cm})$ & Peso Total (g) & Peso (g) & Número nudos & Longitud Total $(\mathrm{cm})$ & Longitud (cm) & Peso (g) & Número \\
\hline Invernadero $(n=41)$ & $29,44(2,62) a$ & $15,80(1,93) \mathrm{a}$ & $7,12(1,32) a$ & $7,36(0,62) a$ & $96,97(8,83) a$ & $8,46(0,58) a$ & $1,26(0,17) a$ & $10,44(0,63) a$ \\
\hline \multirow[t]{3}{*}{ Campo $(n=49)$} & $52,39(2,44) \mathrm{b}$ & $27,84(1,80) b$ & $20,91(1,22) b$ & $17,88(0,57) b$ & $115,73(8,22) a$ & $7,86(0,54) a$ & $1,81(0,15) b$ & $14,02(0,59) \mathrm{b}$ \\
\hline & $F^{*}(p)^{* *}$ & $F(p)$ & $F(p)$ & $F(p)$ & $F(p)$ & $F(p)$ & $F(p)$ & $F(p)$ \\
\hline & $41,04(<, 0001)$ & $20,75(<, 0001)$ & $58,87(<, 0001)$ & $156,68(<, 0001)$ & $2,42(0,1237)$ & $0,57(0,4512)$ & $6,01(0,0162)$ & $17,29(\mathbf{0}, \mathbf{0 0 0 1})$ \\
\hline
\end{tabular}

* Estadístico F. **Probabilidad asociada al ANDEVA, en negrita los valores significativos. Tratamientos cuyas medias tienen letras iguales en una misma columna no difieren ( $p>0,05)$ según el método de medias de mínimos cuadrados.

De forma independiente a la condición de cultivo, el esqueje incrementó los valores de las variables longitud, peso total y del brote emergente, longitud total, promedio y peso de las raíces un $17,64,46,39,50$ y $54 \%$, respectivamente. Con el uso de vitroplantas se incrementó el número de nudos un $21 \%$ en comparación con los esquejes. Por otra parte, solo la longitud total y promedio de las raíces presentaron diferencias significativas $(p \leq, 0001)$ para el factor tipo de explante (Cuadro 2$)$.

En lo que se refiere a los efectos independientes del factor condición de crecimiento respecto al tipo de explante, se presentaron valores mayores para todas las variables medidas en el campo en comparación con el invernadero, excepto para la variable longitud promedio de la raíz, que presentó un aumento no significativo de $7 \%$. La variación del crecimiento de los explantes para la condición campo en relación con el invernadero fue de $44,43,66,59,30$ y $25 \%$ mayor para las variables longitud, peso total y del brote emergente, número de nudos, peso y número de raíces (Cuadro 2).

Para el factor de variación correspondiente a la interacción entre factores (tipo de explante por condición de cultivo), se presentaron diferencias significativas para las variables largo del brote $(F=31,91 ; n=90)$, número de nudos $(F=42,57 ; \mathrm{n}=90)$, número de raíces $(F=10,32 ; \mathrm{n}=90)$, longitud radical total $(F=28,92 ; n=90)$, longitud radical promedio $(F=16,85 ; \mathrm{n}=90)$ y peso de raíces $(F=9,39$; $\mathrm{n}=90)$ (Fig. 4). Solo la variable peso del explante $(F=0,12$; $n=90 ; p=0,7331)$, no presentó diferencias significativas en la interacción entre los factores.

Los valores más altos para las variables longitud del brote, número de nudos y el número de raíces se obtuvieron con la combinación de plantas procedentes del cultivo in vitro cultivadas en condiciones de campo. Por su parte, los explantes procedentes de esquejes y cultivados en condiciones de invernadero presentaron los valores más altos para las variables radiculares (Fig. 4).

Los valores más bajos en el experimento para todas las variables evaluadas se obtuvieron con las plantas procedentes de laboratorio cultivadas en condiciones de invernadero (Fig. 4).

Para el caso de la longitud del brote, las mayores diferencias fueron de 74 y $26 \%$ entre la combinación in vitro - campo respecto a las combinaciones que usaron explantes in vitro $(p<, 0001)$ y esquejes $(p=0,038)$ en la condición invernadero. Las mayores diferencias en cuanto al número de nudos fueron de 73,61 y $38 \%$ entre la combinación in vitro - campo en comparación con los tratamientos in vitro - invernadero, esqueje - invernadero y esqueje - campo $(p<, 0001)$. 

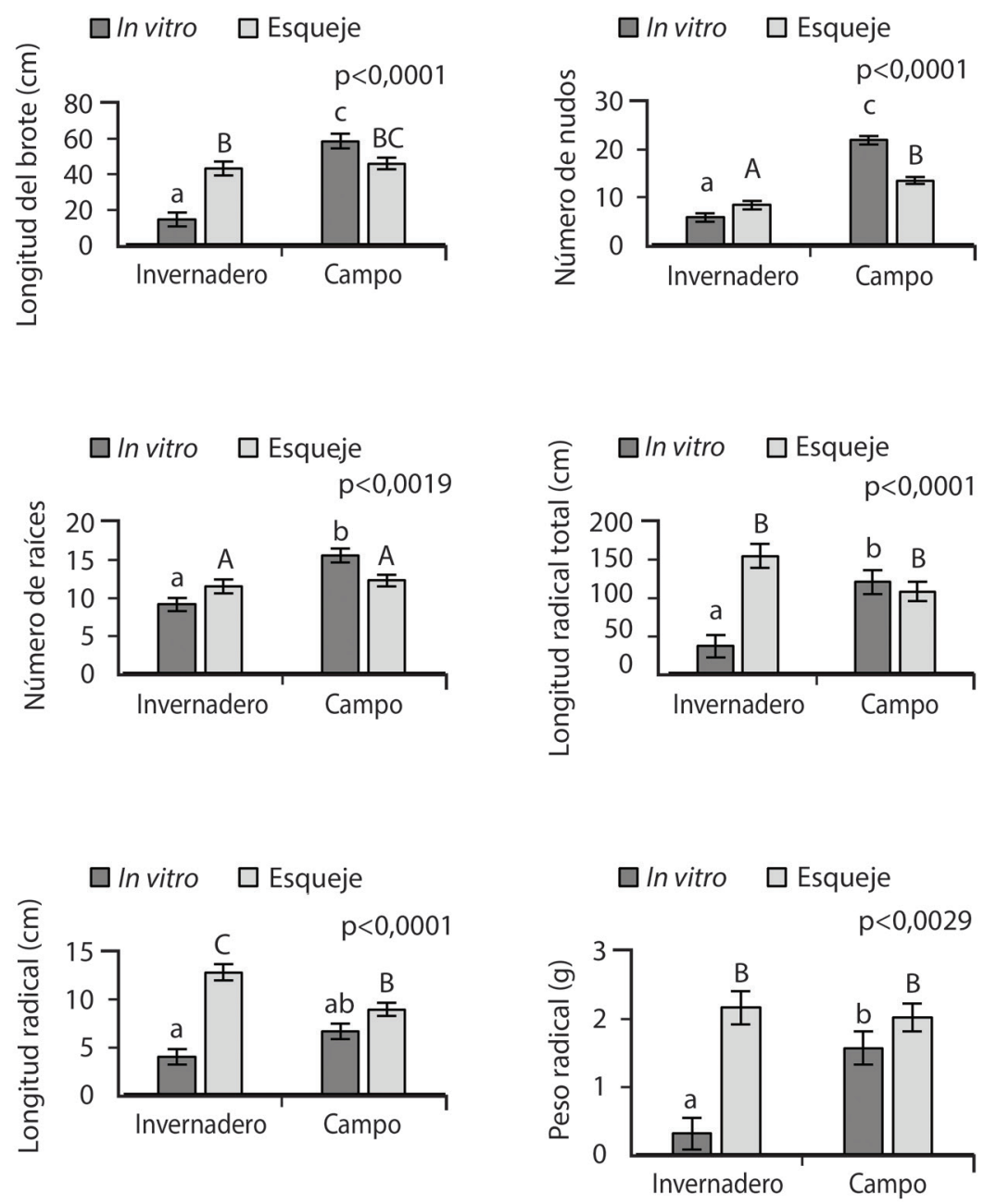

Fig. 4. Respuesta morfogenética de variables de crecimiento de plantas silvestres de V. planifolia según tratamiento de condición de cultivo y tipo de explante. *Letras minúsculas diferentes sobre las barras oscuras indican diferencias significativas entre los tratamientos que evaluaron el crecimiento de plántulas in vitro en condiciones de invernadero y campo, mientras letras mayúsculas diferentes sobre las barras claras indican diferencias significativas entre los tratamientos que evaluaron el crecimiento de los esquejes en ambas condiciones $(\mathrm{p} \leq 0,05)$, según el método de medias de mínimos cuadrados. Probabilidad (Valor $p)$ asociada a la interacción entre factores del ANDEVA.

Los explantes in vitro aumentaron el número de raíces un $40 \%$ cuando se cultivaron en campo respecto a los cultivados en invernadero $(p<, 0001)$ y un 26 y $21 \%$ más raíces que los explantes tradicionales cultivados en invernadero y campo $(p<0,038)$. Por su parte, el alcance radical de las raíces primarias, secundarias y terciarias fue $75 \%$ mayor cuando se cultivó el esqueje tradicional en invernadero respecto a la vitroplanta $(p<, 0001)$ y de 30 y $22 \%$ en comparación con los esquejes y vitroplantas cultivados en campo $(p \geq 0,105)$. Mientras, el largo de la raíz se incrementó 68,48 y $30 \%$ cuando se cultivaron los esquejes en condiciones de invernadero respecto a las vitroplantas cultivadas en invernadero y campo, así como a los esquejes cultivados en campo. El peso radical fue más de 6 veces mayor cuando los esquejes tradicionales se cultivaron en invernadero respecto a las vitroplantas $(p<, 0001)$ pero de solo 27 y $6 \%$ comparado con los esquejes y vitroplantas cultivados en campo ( $p \geq 0,294)$.

\section{DISCUSIÓN}

Ante la falta de información relacionada con el manejo agronómico de individuos silvestres de $V$. planifolia, en condiciones de invernadero y campo a partir de plántulas in vitro y esquejes, se obtuvo un protocolo de manejo que generó información científica básica para conocer la variabilidad de algunos parámetros de crecimiento de forma temporal y espacial; para de esta forma, contribuir a la comprensión de algunos aspectos organogénicos 
fundamentales en cuanto al desarrollo inicial de la especie como cultivo comercial.

Aunque se logró un $100 \%$ de sobrevivencia durante la aclimatización y endurecimiento de las vitroplantas en invernadero, este tipo de explante presentó los valores más bajos para todas las variables en esta condición, lo cual pone en evidencia que en este tipo de estudios, no es conveniente tomar en consideración solo los valores porcentuales de sobrevivencia, pues se incurre en un error al no analizar otras variables relacionadas con la adaptación de esas plantas a condiciones ambientales particulares.

En relación con la longitud del brote lograda en este trabajo $(58,61 \pm 3,93 \mathrm{~cm})$, no hay información en los protocolos de aclimatización que evalúan plantas originadas a partir del cultivo in vitro para ninguna condición de crecimiento durante un periodo como el estudiado (seis meses). Tales trabajos, solo indican valores de supervivencia durante las primeras semanas (Giridhar \& Ravishankar, 2004; Bello-Bello, Martínez-Estrada, Caamal-Velázquez \& Morales-Ramos, 2016; Tan, Chin \& Alderson, 2011; Janarthanam \& Seshadri, 2008; Lee-Espinosa et al., 2008).

Los experimentos encontrados en la literatura con evaluaciones de algunas respuestas morfogénicas, durante el desarrollo inicial del cultivo, muestran diversidad de condiciones a la propuesta en este experimento. A pesar de ello, algunos de estos trabajos han obtenido resultados similares para algunas de las variables analizadas. Por ejemplo, a partir de esquejes con cuatro nudos y cultivados en medios de enraizamiento con proporciones iguales de suelo forestal, estiércol y arena fina, se obtuvo un brote de $57,60 \mathrm{~cm}$, después de 19 semanas en condición de invernadero (Adugna, Belew \& Tilahun, 2015). De forma similar, con la misma cantidad de nudos pero en condiciones de invernadero con suelo forestal y suficiente humus, Hailemichael et al. (2012) obtuvieron un valor máximo de $35,94 \mathrm{~cm}$, después de seis meses. Otros investigadores (Umesha, Murthy \& Smitha, 2011) informaron un valor de $42,4 \mathrm{~cm}$ cuando se cultivaron esquejes de cuatro a cinco nudos en condiciones de invernadero, aunque no indicaron con exactitud el periodo de evaluación para la obtención de ese valor, sin embargo, es congruente con los valores obtenidos en este trabajo para los esquejes cultivados en condiciones de invernadero $(43,47 \pm 3,93 \mathrm{~cm})$ y campo $(46,18 \pm 3,26 \mathrm{~cm})$.

Por su parte, científicos colombianos obtuvieron un brote de $39,25 \mathrm{~cm}$ luego de inocular un sustrato compuesto de fragmentos de madera y vermicompost con hongos endófitos aislados de las raíces de plantas silvestres de vainilla, después de seis meses de evaluación en condiciones de invernadero (Ordóñez, Otero \& Díez, 2012).
Existen otras investigaciones en las cuales se indican valores muy superiores en relación con la longitud de la planta. Al respecto, hay un grupo de trabajo que indicó un valor de $229 \mathrm{~cm}$ luego de siete meses de cultivo en condiciones de vivero, no obstante, fueron experimentos con fertilización química (fórmula: 27-11-11) a una dosis de $20 \mathrm{~g} /$ planta/por año en sustratos compuestos por $75 \%$ de fibra de coco o fragmentos de madera (Osorio, Osorio, Díez \& Moreno, 2014). Además, en la investigación citada, se tomó como variable la altura total de la planta desde la base hasta el ápice de crecimiento y, desde esa perspectiva, no quedó claro si la planta cultivada al inicio de la investigación correspondió a un explante con un ápice de crecimiento activo. De ser así, este meristemo continuará el crecimiento y no se activarán brotes axilares, como ocurrió en este experimento para algunos esquejes (datos no mostrados).

Debido a los resultados obtenidos en cuanto a la generación de nudos por parte de las vitroplantas, es recomendable incluir esta variable como una de las características principales en los estudios que evalúan el desempeño agronómico en las etapas iniciales del cultivo; pues después del tamaño, es el carácter más importante como indicador de producción para los productores de vainilla en México. Esto obedece a una relación empírica entre la mayor cantidad de nudos y la reducción del tiempo de la primera cosecha (Baltazar-Nieto, 2010). Incluso, es propuesto como un indicador adecuado de otras variables dependientes o de respuesta como la longitud total, área foliar, biomasa total y el número de raíces (Arango \& Moreno, 2011).

En lo que respecta al número de raíces, el valor más bajo de este trabajo obtenido a partir de vitroplantas cultivadas en invernadero $(9,27 \pm 0,88 \mathrm{~cm})$, está por encima de los mejores tratamientos de otras investigaciones donde se evaluaron factores diversos. Hailemichael et al. (2012) en las condiciones de cultivo mencionadas obtuvieron 3,68 raíces, mientras que Umesha et al. (2011) solo 1,75 .

Contrario a lo esperado, las diferencias obtenidas en las variables anteriores para las plantas procedentes del cultivo in vitro, refuerzan la hipótesis de la vigorosidad y sanidad de las plantas obtenidas en condiciones asépticas de laboratorio, pero hace falta mucha investigación sobre la plasticidad fenotípica de estos explantes en condiciones naturales para entender si los rasgos observados son medibles y, en qué proporción, están determinados por la genética.

En lo que se refiere a la longitud radical total, el mejor valor obtenido en esta investigación $(155 \pm 15,41 \mathrm{~cm})$ dista de la longitud de $221,97 \mathrm{~cm}$ obtenida por Ordóñez 
et al. (2012) luego de seis meses. El trabajo mencionado, proporcionó valores de referencia de la plasticidad fenotípica de las raíces en su totalidad. Empero, no permite una inferencia adecuada para explicar los datos de esta investigación porque la variación durante el desarrollo y crecimiento radical, son la respuesta a las variadas condiciones ambientales, que se sugiere, son inducidas por la intensidad y variación espacio-temporal en el suministro de agua y nutrientes (Shen et al., 2013).

Ante este escenario, se debe de realizar experimentación controlada para medir dicha plasticidad con estudios sobre: gradientes de intensidad lumínica, humedad, temperatura, riego, diferentes tipos de suelo y sustratos o ambos, y diferentes genotipos, entre otros factores. De esa forma, se podría sugerir un ideotipo de la arquitectura del sistema radical en vainilla para cada parámetro evaluado.

En relación con la longitud de la raíz, el valor más alto obtenido $(12,85 \mathrm{~cm})$ fue muy inferior comparado con los trabajos de Umesha et al. (2011), Hailemichael et al. (2012), y Adugna et al. (2015) quienes obtuvieron valores de 30, 29 y 17,50cm, respectivamente. La presente investigación propone dos procesos que modificaron el crecimiento radicular. Primero, un mecanismo de alargamiento radicular provocado por un estrés nutricional debido al impedimento que ofrecen los tutores artificiales de madera para la absorción de fosforo, por los altos contenidos de hierro (Osorio, 2012). Segundo, un estrés hídrico causado por la superficie de este tipo de tutores al impedir la retención de humedad, provocó un crecimiento continuo de las raíces en búsqueda de agua como mecanismo fundamental de adaptación a condiciones de déficit hídrico (Shao, Chu, Jaleel \& Zhao, 2008). Estos procesos estimularon el desarrollo de raíces adventicias aéreas con longitudes significativamente mayores en los esquejes cultivados en invernadero en contraposición con los de campo (datos no mostrados). En esta última condición, la formación de musgo y líquenes propició microambientes a las raíces aéreas de los esquejes, lo cual evitó la interceptación radicular en el suelo y, por tanto, menores valores de longitud (Fig. 5). Además, sustratos porosos como el utilizado en este estudio, según Osorio (2012), mejoran la absorción de fósforo por la planta, pues la longitud radical es el factor principal en la incorporación de este elemento en la planta.

En algunos ejemplos, cuando las plantas son cultivadas en medios oligotróficos, la relación de partes aéreas/partes subterráneas disminuye, pues las raíces tienden a aumentar la capacidad de absorción (Chen, Mendelssohn, Lorenzen, Brix \& Miao, 2005). Los resultados de este experimento concuerdan con esta afirmación y confirman la condición oligotrófica en invernadero. Además, la planta de vainilla incrementa la demanda nutricional de N, P, K, Ca y Mg, a través del tiempo, para la producción de nuevas células necesarias para el crecimiento, mayor fotosíntesis y vigorosidad del tejido (Monnin \& Perret, 2013). Las menores fluctuaciones de los valores obtenidos para las variables radiculares en la condición de campo para ambos explantes, sugiere poca variación de estos elementos al estar en constante movilización, mientras en invernadero, sin la renovación del sustrato, se generó ambientes de oligotrofia que provocaron mayores fluctuaciones (Fig. 4).

En lo que concierne al peso radical, los valores obtenidos con la combinación esqueje cultivado en vivero $(2,18 \pm 0,24 \mathrm{~g})$ fueron superiores a lo publicado por Adugna et al. (2015), quienes indicaron un valor de 1,16g después de 19 semanas de evaluación. No obstante, muy por debajo del valor de 23,59g obtenido en el trabajo de Hailemichael et al. (2012), el cual es un valor muy elevado teniendo en consideración que la longitud máxima del brote fue solo $36 \mathrm{~cm}$.

Otras causas que podrían explicar la mayor longitud total, promedio y peso radical en los esquejes (en la condición de invernadero) son la poca densidad, buena aireación y drenaje de los sustratos compuestos por musgo, arena y piedra que, indudablemente, incrementaron la porosidad para permitir una mayor penetración y raíces más largas, lo cual está en concordancia con el trabajo de Adugna et al. (2015).

La diversidad de resultados que muestra la literatura en relación con la respuesta morfogenética de estas variables, refleja la inconsistencia metodológica en este tipo de estudios, que buscan el mejoramiento del manejo agronómico en los países productores de vainilla. Al igual que en México, en Costa Rica, muy pocas personas cultivan de forma técnica y adecuada la vainilla no híbrida de forma rentable. Básicamente, no hay estudios sistemáticos para tecnificar el cultivo en planes y programas nacionales de producción. A lo anterior debe agregársele el desconocimiento del material genético de V. planifolia cultivado en el país, así como la calidad productiva y fitosanitaria del mismo. Además, en los últimos años, ha surgido el agravante del cultivo del híbrido de Madagascar, que posee mayores facilidades de manejo, pero no es apto para la exportación a Estados Unidos por el código de regulación federal (21 CFR169.175) que impuso un estándar de identidad para V. planifolia y $V . \times$ tahitensis (FDA, 2016), lo cual impide la comercialización de otras especies con importancia agrícola.

Por último, un error técnico que se ha obviado en todas las investigaciones de vainilla consultadas, es la falta 


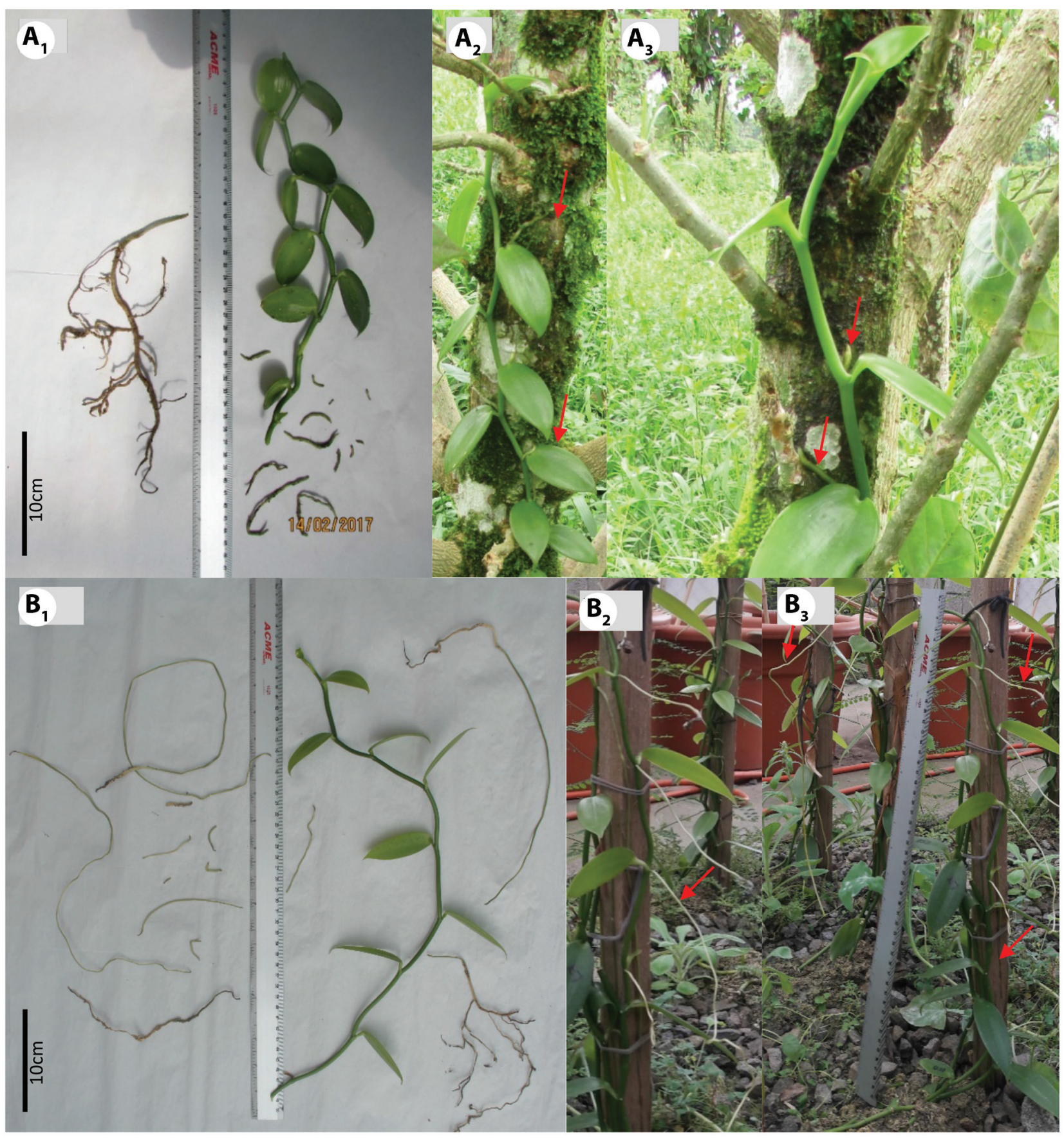

Fig. 5. Diferencias en la longitud de las raíces adventicias del brote nuevo en esquejes cultivados en (A) sistemas agroforestales y (B) en invernadero. Las flechas indican la localización de las raíces de cada explante en ambas condiciones de crecimiento. 
de valores de referencia para la longitud y peso del explante inicial, pues la heterogeneidad de respuestas está muy relacionada con las reservas de carbono de cada explante (Osorio, Osorio, Diez \& Moreno, 2012). Lo anterior es fundamental para el diseño de trabajos afines, pues la variación es afectada por longitudes grandes de poco peso y viceversa, en los explantes originales.

\section{AGRADECIMIENTOS}

Agradecemos al programa de Maestría Académica en Manejo de Recursos Naturales con énfasis en Biodiversidad de la Universidad Estatal a Distancia de Costa Rica porque este trabajo, además de ser requisito de graduación, es parte de los resultados de la tesis de dicho programa.

\section{REFERENCIAS}

Adugna, M., Belew, D., \& Tilahun, D. (2015). Influence of rooting media and number of nodes per stem cutting on nursery performance of vanilla (Vanilla planifolia Andr. syn. Vanilla fragrans). Journal of Horticulture and Forestry, 7(3), 48-56. doi:10.5897/JHF2014.0376

Arango, D. A., \& Moreno, F. (2011). Desarrollo inicial de la vainilla (Vanilla planifolia Andrews, Orchidaceae) bajo diferentes usos de la tierra y condiciones climáticas en Colombia. In I. Lombardi, W. Nalvarte, W. Ojeda, E. Hilario, C. Pajuelo, W. Echeverría, ... J. Saravia (Eds.), Congreso Forestal Latinoamericano pp. 1-17. Lima, Perú: Cámara Nacional Forestal. Recuperado de: http://www. cnf.org.pe/secretaria_conflat/memorias/INDICE.pdf

Azofeifa-Bolaños, J. B., Gigant, L. R., Nicolás-García, M., Pignal, M., Tavares-González, F. B., Hágsater, E., ... Grisoni, M. (2017). A new vanilla species from Costa Rica closely related to V. planifolia (Orchidaceae). European Journal of Taxonomy, 284, 1-26. doi:10.5852/ejt.2017.284

Azofeifa-Bolaños, J. B., Paniagua-Vásquez, A., \& García-García, J. A. (2014). Importancia y desafíos de la conservación de Vanilla spp. (Orchidaceae) en Costa Rica. Agronomía Mesoamericana, 25(1), 189-202. doi:10.15517/ am.v25i1.14220

Baltazar-Nieto, P. (2010). Caracteres morfológicos de vainilla utilizados por el agricultor en la selección del material reproductivo en cuatro municipios del Totonacapan, México. Colegio de Postgraduados,México.

Belanger, F.C., \& Havkin-Frenkel, D. (2011). Molecular analysis of a Vanilla hybrid cultivated in Costa Rica. In: D. Havkin-Frenkel, \& F.C. Belanger. (Eds), Handbook of Vanilla Science and Technology pp. 256- 263. Wiley-Blackwell, Chichester, United Kingdom. doi:0.1002/9781444329353
Bello-Bello, J. J., Martínez-Estrada, E., Caamal-Velázquez, J. H., \& Morales-Ramos, V. (2016). Effect of LED light quality on in vitro shoot proliferation and growth of vanilla (Vanilla planifolia Andrews). African Journal of Biotechnology, 15(8), 272-277. doi:10.5897/AJB2015.14662

Chen, H., Mendelssohn, I. A., Lorenzen, B., Brix, H., \& Miao, S. (2005). Growth and nutrient responses of Eloecharis cellulosa (Cyperaceae) to phosphate level and redox intensity. American Journal of Botany, 92(9), 1457-1466. doi:10.3732/ajb.92.9.1457

FDA (Food and Drug Administration). (2017). Food Dressings and Flavorings. Recuperado de: https://www.accessdata.fda.gov/scripts/cdrh/cfdocs/cfcfr/CFRSearch.cfm?CFRPart $=169 \&$ showFR=1\&subpartNode $=21: 2.0 .1 .1 .44 .1$

Giridhar, P., \& Ravishankar, G. A. (2004). Efficient micropropagation of Vanilla planifolia Andr. under influence of thidiazuron, zeatin and coconut milk. Indian Journal of Biotechnology, 3, 113-118.

Gómez, N. (2012). Respuestas de Vanilla planifolia Jacks. ante variaciones microambientales bajo arreglos agroforestales en un bosque seco tropical. Universidad Nacional de Colombia. Recuperado de: http://www.bdigital.unal. edu.co/8273/1/43455134.pdf

Hailemichael, G., Tilahun, D., Kifelw, H., \& Mitiku, H. (2012). The effect of different node number cuttings on nursery performance of Vanilla (Vanilla planifolia syn. Vanilla fragrans) in south western Ethiopia. International Research Journal of Agricultural Science and Soil Science, 2(9), 408-412.

IMN. (2011). Promedios mensuales de datos climáticos Estación Automática Finca Diamantes, Guápiles, Limón. Gestión de Información y Comercialización. San José, Costa Rica.

Janarthanam, B., \& Seshadri, S. (2008). Plantlet regeneration from leaf derived callus of Vanilla planifolia Andr. In Vitro Cellular \& Developmental Biology - Plant, 44(2), 84-89. doi:10.1007/s11627-008-9123-4

Lee-Espinosa, H. E., Murguía-González, J., García-Rosas, B., Córdova-Contreras, A. L., Laguna-Cerda, A., MijangosCortés, J. O., ... \& Santana-Buzzy, N. (2008). In vitro clonal propagation of vanilla (Vanilla planifolia "Andrews"). HortScience, 43(2), 454-458.

Monnin, M. E., \& Perret, J. (2013). Absorción de los nutrientes y crecimiento de la vainilla (Vanilla planifolia). Tierra Tropical, 9(1), 29-37. Recuperado de: http://tierratropical.org/es/editions/issue-9-1-2013/absorption-of-nutrients-and-growth-of-vanilla-vanilla-planifolia/

Ordóñez, N. F., Otero, J. T., \& Díez, M. C. (2012). Hongos endófitos de orquídeas y su efecto sobre el crecimiento en Vanilla planifolia Andrews. Acta Agronómica, 61(3), 282-290.

Osorio, A. (2012). Efecto de materiales orgánicos, fertilizantes e inóculos microbiales sobre el crecimiento y nutrición de plántulas de vainilla (Vanilla planifolia Jacks). Universidad Nacional de Colombia. 
Osorio, A. I., Osorio, N. W., Díez, M. C., \& Moreno, F. (2014). Nutrient status and vegetative growth of Vanilla planifolia Jacks plants as affected by fertilization and organic substrate composition. Acta Agronómica, 63(4), 326334. doi:10.15446/acag.v63n4.40754

Osorio, A. I., Osorio, N. W., Diez, M. C., \& Moreno, F. H. (2012). Effects of organic substrate composition, fertilizer dose, and microbial inoculation on vanilla plant nutrient uptake and growth. Acta Horticulturae, 964, 135-142. doi:10.17660/ActaHortic.2012.964.17

Quesada, R. (2007). Los Bosques de Costa Rica. In Instituto Tecnológico de Costa Rica (Ed.), IX Congreso Nacional de Ciencias Exploraciones: Exploraciones fuera y dentro del aula pp. 1-16. Cartago, CR: Instituto Tecnológico de Costa Rica.

Shao, H. B., Chu, L. Y., Jaleel, C. A., \& Zhao, C. X. (2008). Waterdeficit stress-induced anatomical changes in higher plants. Comptes Rendus - Biologies, 331(3), 215-225. doi:10.1016/j.crvi.2008.01.002

Shen, J., Li, C., Mi, G., Li, L., Yuan, L., Jiang, R., \& Zhang, F. (2013). Maximizing root/rhizosphere efficiency to improve crop productivity and nutrient use efficiency in intensive agriculture of China. Journal of Experimental Botany, 64(5), 1181-1192. doi:10.1093/jxb/ers342

Soto-Arenas, M. A. (1999). Filogeografía y recursos genéticos de las vainillas de México. México, D. F.

Soto-Arenas, M. A., \& Solano-Gómez, A. R. (2007). Ficha técnica de Vanilla planifolia. In M. A. Soto-Arenas (Ed.), Información actualizada sobre las especies de orquídeas del PROY-NOM-059-ECOL- 2000. (pp. 1-18). México, D. F.: Instituto Chinoin A.C., Herbario de la Asociación Mexicana de Orquideología A.C. Bases de datos SNIBCONABIO. Proyecto No. W029. Recuperado de: http:// www.conabio.gob.mx/conocimiento/ise/fichasnom/ Vanillaplanifolia00.pdf

Tan, B. C., Chin, C. F., \& Alderson, P. (2011). An Improved Plant Regeneration of Vanilla planifolia Andrews. Plant Tissue Culture and Biotechnology, 21(1), 27-33.

Umesha, K., Murthy, G., \& Smitha, G. R. (2011). Environmental conditions and type of cuttings on rooting and growth of vanilla (Vanilla planifolia Andrews). Journal of Tropical Agriculture, 49(1-2), 121-123. Recuperado de: http:// jtropag.kau.in/index.php/ojs2/article/view/251/251 